\title{
Latest results from NEMO-3 and status of SuperNEMO
}

\author{
Remoto Alberto*i \\ on behalf of NEMO-3 and SuperNEMO collaborations \\ Laboratoire d'Annecy le Vieux de physique des particules, 9 Chemin de Bellevue 74941 \\ Annecy-le-Vieux CEDEX FRANCE \\ E-mail: remotodin2p3.fr
}

\begin{abstract}
The NEMO-3 experiment performed precise measurement of the double beta decay and searched for the neutrino-less double beta decay on seven isotopes, among which ${ }^{100} \mathrm{Mo}$ and ${ }^{82} \mathrm{Se}$ were the dominant ones. The detector, installed in the Laboratoire Souterrain de Modane, took data for 8 years before being decommissioned in 2011. The key feature of NEMO-3 was its unique capability to fully reconstruct the kinematics of the events, which allowed to reduce the backgrounds and to discriminate among different mechanisms beyond the neutrino-less double beta decay. No evidence of $0 v \beta \beta$ has been found with an exposure of $34.3 \mathrm{~kg} \times \mathrm{y}$ of ${ }^{100} \mathrm{Mo}$, providing a limit for the light Majorana neutrino mass mechanism of $T_{1 / 2}^{0 v \beta \beta}>1.1 \times 10^{24} \mathrm{y}(90 \%$ C.L.) which corresponds to an effective neutrino mass of $\left|m_{\beta \beta}\right|<0.33-0.62 \mathrm{eV}$, depending from the NME considered. Furthermore, no background events in the double electron channel has been found in the energy region $3.2-10 \mathrm{MeV}$ after an exposure of $47 \mathrm{~kg} \times \mathrm{y}$. The same experimental technique is adopted for the next generation experiment called SuperNEMO. The new detector has a modular design with the capability to measure different isotopes at the same time. ${ }^{48} \mathrm{Ca},{ }^{82} \mathrm{Se}$ and ${ }^{150} \mathrm{Nd}$ are currently under consideration. With 20 detection modules observing for 5 years $100 \mathrm{~kg}$ of ${ }^{82} \mathrm{Se}$, the expected sensitivity should reach $T_{1 / 2}^{0 v}>10^{26}$ y $\left(\left|m_{\beta \beta}\right|<0.04-0.10 \mathrm{eV}\right)$, competitive with other experiments. In order to demonstrate the feasibility of the full experiment, the first step is the imminent construction of a first demonstrator module containing $7 \mathrm{~kg}$ of ${ }^{82} \mathrm{Se}$. With an expected sensitivity of $T_{1 / 2}^{0 v}>6.6 \times 10^{24} \mathrm{y}\left(\left|m_{\beta \beta}\right|<0.2-0.4 \mathrm{eV}\right)$ after $2.5 \mathrm{y}$, the demonstrator module will also be able to perform a competitive measurement.
\end{abstract}

XVI International Workshop on Neutrino Telescopes,

2-6 March 2015

Palazzo Franchetti âĂŞ Istituto Veneto, Venice, Italy

\footnotetext{
*Speaker.

${ }^{\dagger}$ Supported by the Labex ENIGMASS, Investissements d’Avenir.
} 


\section{Introduction}

Experimental searches for neutrino-less double beta decay $(0 v \beta \beta)$ are one of the most active research topics in neutrino physics. The observation of such process is in fact of major importance since it will prove the Majorana nature of neutrinos and may give access to their absolute mass scale. The Majorana nature of the neutrino would have interesting implication in many extensions of the Standard Model of particle physics. For instance the see-saw mechanism requires the existence of a Majorana neutrino to explain naturally the smallness of neutrino masses. A Majorana neutrino would also provide a natural framework for the lepton number violation, and particularly for the leptogenesis process which may explain the observed matter-antimatter asymmetry of the Universe. The standard underlying mechanism providing a neutrino-less double beta decay is the exchange of a Majorana neutrino. In this case, the decay half-life can be written as:

$$
\left[T_{0 v}(A, Z)\right]^{-1}=G_{0 v}\left(Q_{\beta \beta}, Z\right)\left|M_{0 v}(A, Z)\right|^{2}\left|m_{\beta \beta}\right|^{2}
$$

where $\left|m_{\beta \beta}\right|$ is the effective neutrino mass, $M_{0 v}$ is the Nuclear Matrix Element (NME) and $G_{0 v}$ is the kinematical phase space factor. Other mechanisms could be involved in this process as the existence of right handed currents in the electroweak interaction, the exchange of super symmetric particles via the R-parity violating coupling, or extra dimensions and would results in a different topology of the final state. For a given mechanism and isotope, the $0 v \beta \beta$ decay half-life depends on the phase space factors and on the NME. While the former can be precisely calculated [1], the latter depends on the nuclear model adopted [2]. The decay half-lives of different isotopes can then differ by a few orders of magnitude with large theoretical uncertainties from NME calculations. Furthermore, the quenching of the axial coupling constant $g_{A}$ observed in single $-\beta$ and $2 v \beta \beta$ decay could increase half-lives $0 v \beta \beta$ as discussed in [3]. It is therefore essential to search for $0 v \beta \beta$ decays in several isotopes.

\section{The NEMO-3 experiment}

The NEMO-3 detector, shown in Fig. 1 (a), performed precise measurement of double beta decay and searched neutrino-less double beta decay on seven isotopes, among which ${ }^{100} \mathrm{Mo}(\sim 7 \mathrm{~kg})$ and ${ }^{82} \mathrm{Se}(\sim 1 \mathrm{~kg})$. Smaller amount of other isotopes as ${ }^{130} \mathrm{Tl},{ }^{116} \mathrm{Cd},{ }^{150} \mathrm{Nd},{ }^{96} \mathrm{Zr}$ and ${ }^{48} \mathrm{Ca}$ were also present, Fig. 1 (b). The detector [4] had a cylindrical symmetry and was divided in 20 sectors. Each sector consisted in source planes containing the $\beta \beta$ emitting isotopes surrounded by a tracker and a calorimeter. The detector was installed in the Laboratoire Souterrain de Modane (LSM), under a rock overburden of 4800 m.w.e and took data between 2003 and 2011. The $\beta \beta$ events were emitted from thin source foils $\left(40-60 \mathrm{mg} / \mathrm{cm}^{2}\right)$ suspended between two concentric cylindrical tracking volumes. The tracker was composed by 6180 Geiger cells allowing a tracking precision of $\sigma_{t}=2-3 \mathrm{~mm}$ transversally to the cells and $\sigma_{z}=7-13 \mathrm{~mm}$ longitudinally. The tracking volume was surrounded by a segmented calorimeter made of 1940 large blocks of plastic scintillator coupled to low radioactivity 3" and 5" PMTs. The calorimeter provided a timing resolution of $\sigma=250$ ps while the energy resolution was $14-17 \%$ FWHM for $1 \mathrm{MeV}$ electrons. The detector was immersed in a $25 \mathrm{G}$ magnetic field to enhance charged particle identification, shielded from external gamma rays by $19 \mathrm{~cm}$ of low activity iron and from neutrons by $30 \mathrm{~cm}$ of water with boric 


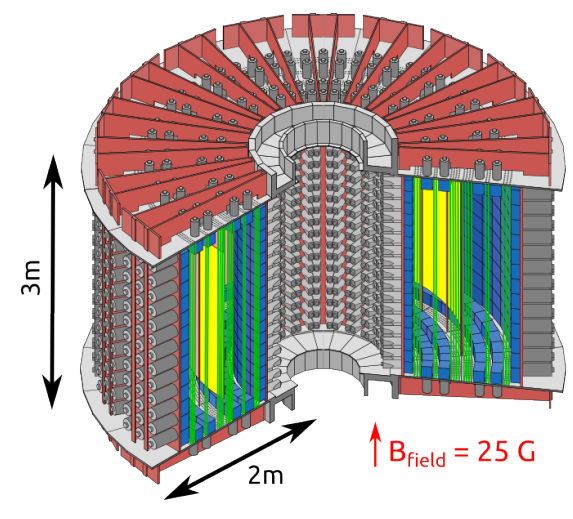

(a)

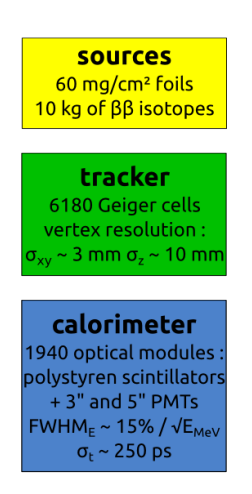

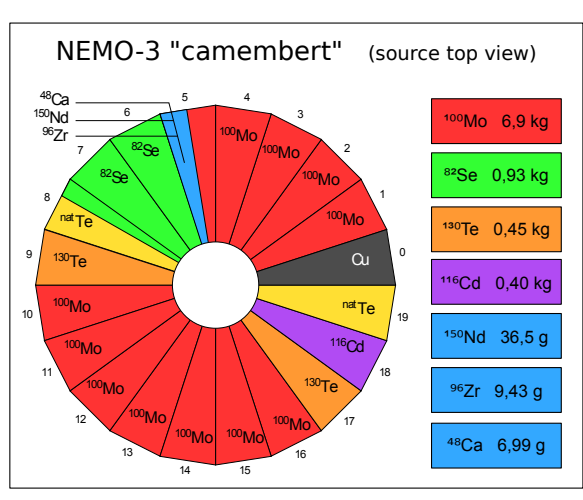

(b)

Figure 1: (a) Schematic view of the NEMO-3 detector without external shielding. (b) Distribution of the $\beta \beta$ source isotopes in the NEMO-3 sectors.

acid. After one year of data taking, a radon-free air facility flushing a tight tent surrounding the detector was installed to reduce the radon contamination in the tracking chamber by a factor 6 . The data are then divided in two data taking period, namely Phase I (before the anti-radon facility installation) with a live time of $1.02 \mathrm{y}$ and Phase II (after the anti-radon facility installation) with a live time of $3.94 \mathrm{y}$.

The detector energy calibration has been performed introducing up to 60 radioactive sources in the detector through twenty calibration tubes located near the source foils. The absolute energy scale calibration was performed every 3 weeks with ${ }^{207} \mathrm{Bi}$ sources which provide internal conversion electrons of $482 \mathrm{keV}$ and $976 \mathrm{keV}$. For $99 \%$ of the PMTs the energy scale is known better than $2 \%$. The rare $1682 \mathrm{keV}$ internal conversion electron peak of ${ }^{207} \mathrm{Bi}$ was used to determine the systematic uncertainty on the energy scale which was found to be within $0.2 \%$. The energy scale was also tested with the end-point of the $\beta$ spectrum of ${ }^{90} \mathrm{Y}$ sources $\left(Q_{\beta}=2.28 \mathrm{MeV}\right)$ and of ${ }^{214} \mathrm{Bi}\left(Q_{\beta}=3.27 \mathrm{MeV}\right)$ from ${ }^{222} \mathrm{Rn}$ in the tracker volume. The linearity of the PMTs was verified with light injection test during the construction phase and deviation was found to be within $1 \%$ in $0-4 \mathrm{MeV}$ energy range. The relative gain and time variation of individual PMTs were then surveyed twice a day by a light injection system. PMTs that show a gain variation above $5 \%$ compared to a linear interpolation between two successive absolute calibrations with ${ }^{207} \mathrm{Bi}$ are excluded from the analysis.

The main feature of NEMO-3 was its unique capability to fully reconstruct the kinematics of the events as shown in Fig. 2. This allows to perform topological selection of the events depending from the final states of interest, to reduce backgrounds and to discriminate among different mechanisms beyond the neutrino-less double beta decay [5].

\subsection{Background measurements}

The most important backgrounds for a $\beta \beta$ decay experiment come from the natural radioactivity of the detector materials due to the presence of long half-life isotopes, mainly ${ }^{238} \mathrm{U},{ }^{235} \mathrm{U},{ }^{232} \mathrm{Th}$ and ${ }^{40} \mathrm{~K}$ and their decay products. Since NEMO-3 detector was capable to identify different type of particles and event topologies, it has been possible to study the backgrounds combining tracking, 

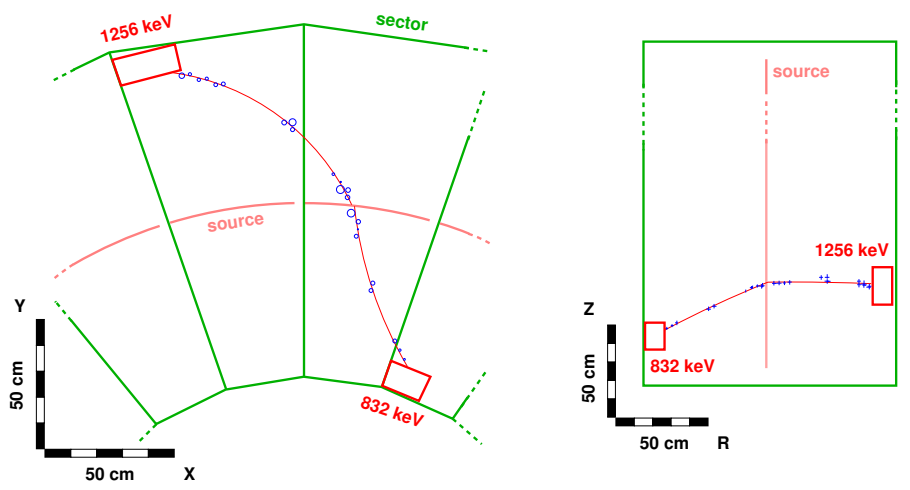

Figure 2: Top (left) and side (right) views of a $\beta \beta$ event detected in NEMO-3. Two tracks are reconstructed from a single vertex in the source foil, with a negative (electron-like) curvatures in the magnetic field, and associated to energy deposition in two calorimeter blocks.

calorimetric and timing information in different channels [6]. Activities of different background components are then obtained by adjusting Monte Carlo (MC) distributions to the data in different channels. The external backgrounds produced by the $\gamma$-ray flux entering the detector is determined by the detection of the electron produced by Compton effect in one of the scintillators, which cross the entire detector, as shown in Fig. 3 (a) and (b). The internal backgrounds due to contamination in the source foil is quantified looking for single $\beta$ emission, as shown in Fig. 3 (c), or for $\beta-n \gamma$, if one or several $\gamma$ are emitted together with the electron. The radon contribution is determined studying the delayed $\beta-\alpha$ events produced by the ${ }^{214} \mathrm{Bi} \rightarrow{ }^{214} \mathrm{Po}$ cascade of ${ }^{222} \mathrm{Rn}$ chain.

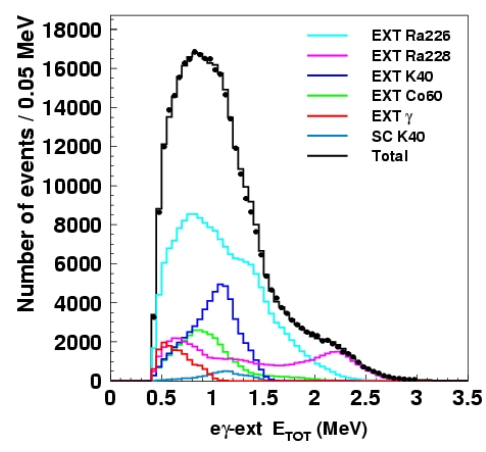

(a)

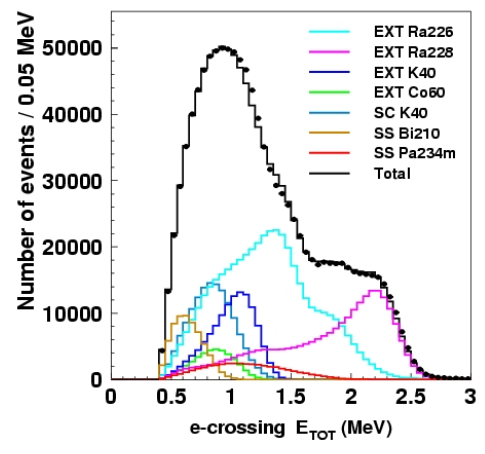

(b)

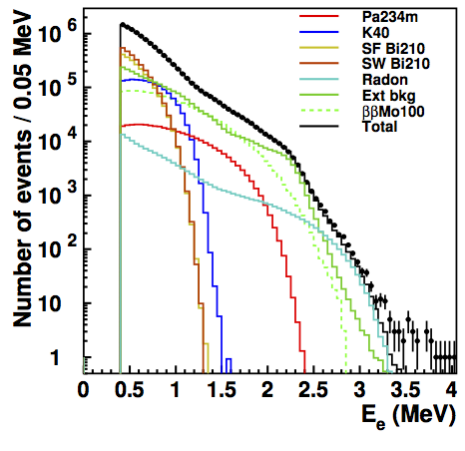

(c)

Figure 3: Fit of the energy distributions of external backgrounds in $1 e^{-} 1 \gamma$ channel (a) and one crossing electron channel (b). Fit of the internal backgrounds in $1 e^{-}$channel (c).

The background model developed for the $0 v \beta \beta$ search in the ${ }^{100}$ Mo has been validated performing several tests. The ${ }^{208} \mathrm{Tl}$ activity was validated with two calibrated ${ }^{232} \mathrm{U}$ sources (decaying to ${ }^{208} \mathrm{Tl}$ ) which provide a $10 \%$ systematics uncertainty compared to HPGe activity measurements of the radioactive source. The ${ }^{214} \mathrm{Bi}$ activity was validated comparing the measurement obtained in the $1 e^{-} 1 \alpha$ and the $1 e^{-} N \gamma$ channels and have been found in agreement within $10 \%$ systematics. The contribution from these contaminations in the $2 e$ channel was then tested independently. The ${ }^{208} \mathrm{Tl}$ 
background was tested in the $2 e^{-} N \gamma$ channel where 7 events were observed in the $2.8-3.2 \mathrm{MeV}$ energy region for 8.8 expected, as shown in Fig. 4 (a). The ${ }^{214} \mathrm{Bi}$ background was tested in the $2 e^{-} \alpha$ channel where 3 events were observed for $6.5 \pm 0.4$ expected in Phase I and 3 events observed for $2.9 \pm 0.2$ expected in Phase II. The background model was finally tested selecting $2 e^{-}$ events from the copper and tellurium foils where no double beta decay events are expected at high energy $\left(Q_{\beta \beta}\left({ }^{130} \mathrm{Te}\right)=2.53 \mathrm{MeV}\right)$. A very good agreement has been obtained with an exposure of $13.5 \mathrm{~kg} \times \mathrm{y}$, as shown in Fig. 4 (b). It is worth to notice that no events have been observed above $3.1 \mathrm{MeV}$.

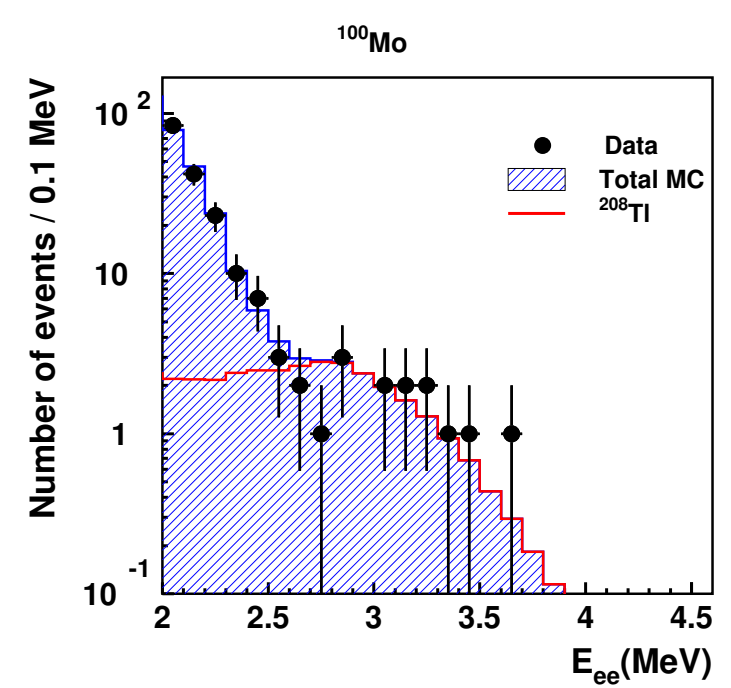

(a)

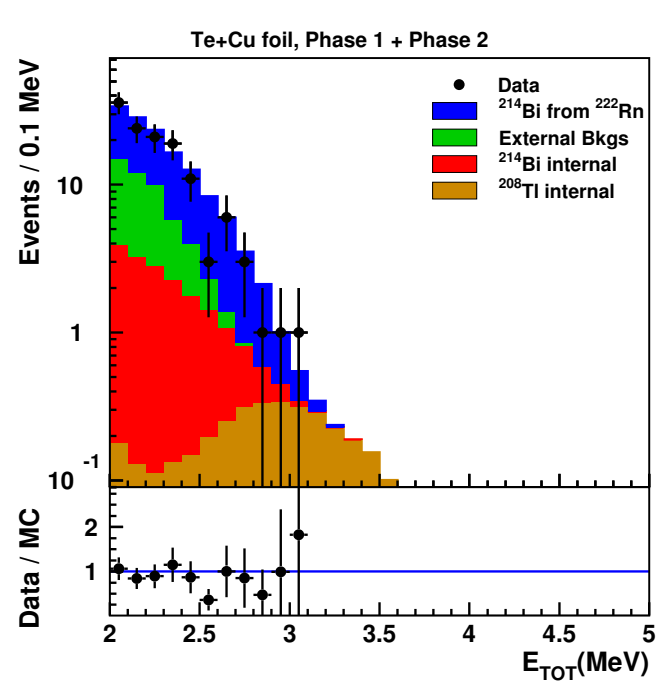

(b)

Figure 4: (a) Validation of the ${ }^{208} \mathrm{Tl}$ measurement in the $2 e^{-} N \gamma$ channel. (b) Validation of the background model in the $2 e^{-}$channel from the copper and tellurium foils where no double beta decay events are expected.

\subsection{Measurements of $2 v \beta \beta$ in ${ }^{100} \mathrm{Mo}$}

The $2 v \beta \beta$ decay represents the irreducible background for the $0 v \beta \beta$ searches. The measurement of the $2 v \beta \beta$ is however interesting since it may provide information for the theoretical calculation of the NME and tests different nuclear models [7]. The analysis of the Phase I data provide the most precise direct measurement of the ${ }^{100} \mathrm{Mo} 2 v \beta \beta$ decay half-life corresponding to $T_{1 / 2}^{2 v}=7.16 \pm 0.01($ stat $) \pm 0.54($ syst $) \times 10^{18} \mathrm{y}$ [8]. More recently the analysis of Phase I and Phase II results in $T_{1 / 2}^{2 v}=6.93 \pm 0.04($ stat $) \times 10^{18} \mathrm{y}$ for $E_{2 e^{-}}>2 \mathrm{MeV}$ [9] . This value is in agreement with the previously published result and with the word average [10].

Thanks to the detector capability to reconstruct single particles, NEMO-3 was not only able to measure the summed electron energy spectrum but also the single electron energy and the angle between the two electrons as shown in Fig. 5.

\subsection{Search for $0 v \beta \beta$ in ${ }^{100} \mathrm{Mo}$}

The search for $0 v \beta \beta$ decay for ${ }^{100}$ Mo has been performed on the total accumulated expo- 

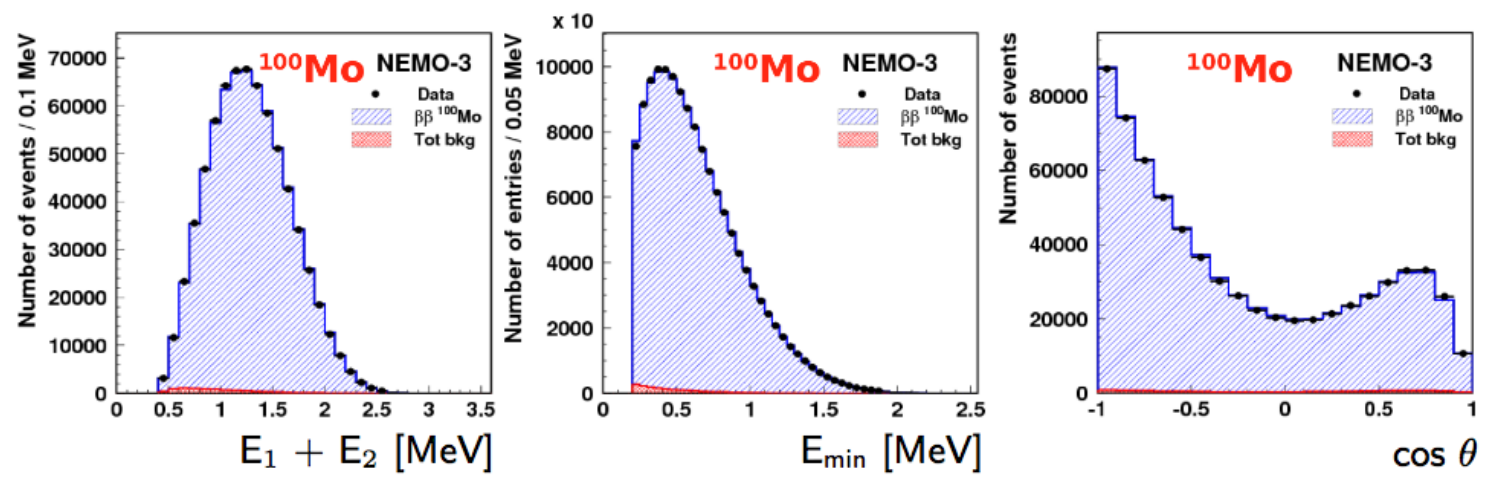

Figure 5: Total energy (left), individual energy (center) and angular distributions (right) of the ${ }^{100}$ Mo $2 v \beta \beta$ events in Phase II.

sure of $34.3 \mathrm{~kg} \times \mathrm{y}$ [9]. The search has been focused in the $2.8-3.2 \mathrm{MeV}$ energy window, around the $Q_{\beta \beta}$ value of ${ }^{100} \mathrm{Mo}$. In this energy window the signal detection efficiency is $4.7 \%$. The background contributions obtained from the measurements presented in the previous sections are summarised in Tab. 1.

\begin{tabular}{|l|c|c|c|}
\hline Data sets & $\begin{array}{c}\text { Phase } 1 \\
7.1 \mathrm{~kg} \times \mathrm{y}\end{array}$ & $\begin{array}{c}\text { Phase } 2 \\
27.2 \mathrm{~kg} \times \mathrm{y}\end{array}$ & $\begin{array}{c}\text { Combined } \\
34.3 \mathrm{~kg} \times \mathrm{y}\end{array}$ \\
\hline External background & $<0.04$ & $<0.16$ & $<0.2$ \\
${ }^{214} \mathrm{Bi}$ from ${ }^{222} \mathrm{Rn}$ & $2.8 \pm 0.3$ & $2.5 \pm 0.2$ & $5.2 \pm 0.5$ \\
${ }^{214} \mathrm{Bi}$ internal & $0.20 \pm 0.02$ & $0.80 \pm 0.08$ & $1.0 \pm 0.1$ \\
${ }^{208} \mathrm{Tl}$ internal & $0.65 \pm 0.05$ & $2.7 \pm 0.2$ & $3.3 \pm 0.3$ \\
$2 v 2 \beta$ & $1.28 \pm 0.02$ & $7.16 \pm 0.05$ & $8.45 \pm 0.05$ \\
Total expected & $4.9 \pm 0.3$ & $13.1 \pm 0.3$ & $18.0 \pm 0.6$ \\
\hline Data & 3 & 12 & 15 \\
\hline
\end{tabular}

Table 1: Expected background and observed $2 e^{-}$events in NEMO-3 after a $34.3 \mathrm{~kg} \times \mathrm{y}$ exposure with ${ }^{100} \mathrm{Mo}$ in the energy range $2.8-3.2 \mathrm{MeV}$.

No event excess has been observed above the background expectation and a limit for the halflife of $0 v \beta \beta$ decay is obtained using a modified frequentist analysis based on log-likelihood ratio test statistics [11]. Dedicated studies estimate the main systematic uncertainties to be $7 \%$ for the detection efficiency determined using dedicated runs with activity-calibrated ${ }^{207} \mathrm{Bi}$ sources. The uncertainty on $2 v \beta \beta$ is obtained from the fit to $2 e^{-}$events above $2 \mathrm{MeV}$ and is $0.7 \%$. The uncertainty on the ${ }^{214} \mathrm{Bi}$ and ${ }^{208} \mathrm{Tl}$ are estimated as described in the previous section and are found to be $10 \%$ respectively. The information of the binned energy sum distribution in the $2.0-3.2 \mathrm{MeV}$ energy window is used for signal and background (Fig. 6) together with the statistical and systematic uncertainties and their correlations as described in [11]. The background-only hypothesis is accepted with a p-value of $64.7 \%$. The half-life limit for the neutrino mass mechanism is $T_{1 / 2}^{0 v}>1.1 \times 10^{24}$ y $\left(90 \%\right.$ C.L.), which corresponds to $\left|m_{\beta \beta}\right|<0.33-0.62 \mathrm{eV}$, depending on the NME [2] and the phase space calculations [1].

Limits on other lepton number violating mechanisms have been also obtained in [9] and are sum- 


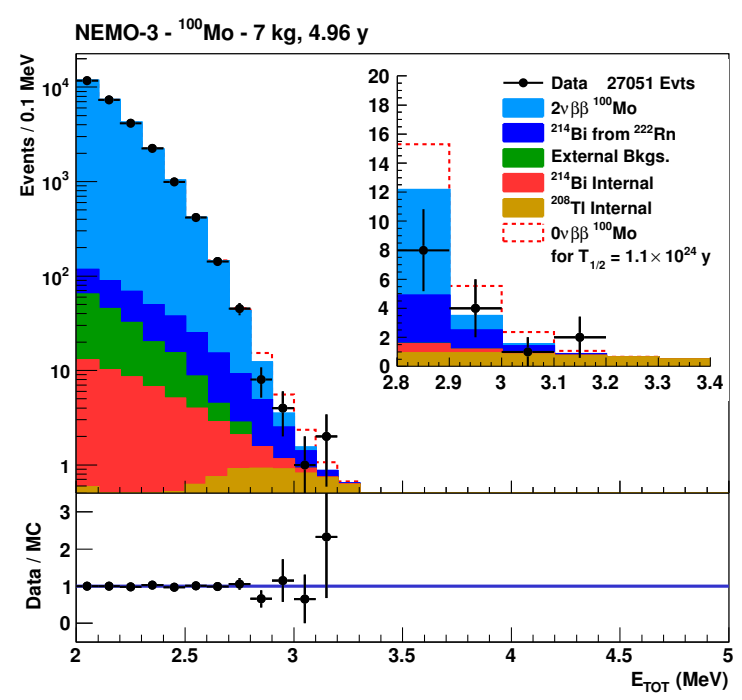

Figure 6: Distribution of the $2 e^{-}$energy sum of ${ }^{100} \mathrm{Mo}$ above $2 \mathrm{MeV}$ and ratio between the observed and the expected distributions from MC simulations [9].

marised in Tab. 2. Right-left symmetric models can produce $0 v \beta \beta$ decay via right-handed currents in the electroweak Lagrangian. This mechanism leads to different angular and single energy distributions of the final state electrons and can therefore be distinguished in a NEMO-like experiment [5]. The corresponding half-life limits translate into an upper bound on the coupling between righthanded quark and lepton currents of $\langle\lambda\rangle<(0.9-1.3) \times 10^{-6}(90 \%$ C.L.) or between right-handed quark and left-handed lepton currents of $\langle\eta\rangle\left\langle(0.5-0.8) \times 10^{-8}\right.$ (90\% C.L.). The constraints are obtained using the NME calculations from [15]. In supersymmetric models the $0 v \beta \beta$ process is mediated by the exchange of a gluino or a neutralino. Using the above half-life limit and the NME from [16] an upper bound is obtained on the trilinear $R$-parity violating supersymmetric coupling of $\lambda_{111}^{\prime}<(4.4-6.0) \times 10^{-2} \mathrm{f}$, where $\mathrm{f}=\left(\mathrm{M}_{\tilde{\mathrm{q}}} / 1 \mathrm{TeV}\right)^{2}\left(\mathrm{M}_{\tilde{\mathrm{g}}} / 1 \mathrm{TeV}\right)^{1 / 2}$ and $\mathrm{M}_{\tilde{\mathrm{q}}}$ and $\mathrm{M}_{\tilde{\mathrm{g}}}$ represent the squark and the gluino masses. Finally, the $0 v \beta \beta$ decay can be accompanied by the emission of a Majoron, a light or massless boson that could weakly couple to the neutrino. The energy sum of the two emitted electrons will have a broad spectrum ranging from zero to the $Q_{\beta \beta}$ of ${ }^{100} \mathrm{Mo}$. The exact shape will depend on the spectral index $n$, which determines the phase space dependence on the energy released in the decay, $\mathrm{G}^{0 v} \propto\left(\mathrm{Q}_{\beta \beta}-\mathrm{E}_{\mathrm{TOT}}\right)^{n}$. The lower bound on the half-life of the Majoron accompanied $0 v \beta \beta$ decay with the spectral index $n=1$ is $0.027 \times 10^{24} \mathrm{y}$. This limit is almost a factor of two more stringent than the previous best limit for this isotope [17]. Taking into account the phase space factors given in [18] and the NME calculated in [2], an upper bound on the Majoron-neutrino coupling constant has been obtained, $\left\langle g_{e e}\right\rangle<(1.6-3.0) \times 10^{-5}$. All results obtained for the different mechanisms by NEMO-3 are comparable with the best current results obtained with other isotopes.

It is worth to notice that no events have been observed in the $3.2-10 \mathrm{MeV}$ energy window for NEMO-3 sources containing isotopes with $Q_{\beta \beta}$ lower than $3.2 \mathrm{MeV}$ (i.e. ${ }^{100} \mathrm{Mo},{ }^{82} \mathrm{Se},{ }^{130} \mathrm{Te}$ and ${ }^{116} \mathrm{Cd}$ ) or without $\beta \beta$ emitters $(\mathrm{Cu})$ during the entire running period, which corresponds to an exposure of $47 \mathrm{~kg} \times \mathrm{y}$. The tracking+calorimetry technique adopted by NEMO-3 may allow to perform 


\begin{tabular}{|l|c|c|c|}
\hline $0 v \beta \beta$ Process & Statistical only & With systematics & $\begin{array}{c}\text { Expected with systematics } \\
\text { (median and } \pm 1 \sigma \text { range) }\end{array}$ \\
\hline Mass mechanism & 1.1 & 1.1 & $1.0[0.7-1.4]$ \\
RH current $\langle\lambda\rangle$ & 0.7 & 0.6 & $0.5[0.4-0.8]$ \\
RH current $\langle\eta\rangle$ & 1.0 & 1.0 & $0.9[0.6-1.3]$ \\
Majoron & 0.050 & 0.044 & $0.039[0.027-0.059]$ \\
\hline
\end{tabular}

Table 2: $90 \%$ C.L. limits on the half-lives of lepton number violating processes (in units of $10^{24} \mathrm{y}$ ).

background free $0 v \beta \beta$ searches for isotopes with high $Q_{\beta \beta}$ such as ${ }^{48} \mathrm{Ca},{ }^{96} \mathrm{Zr}$ or ${ }^{150} \mathrm{Nd}$.

\subsection{Results with other isotopes}

The $\beta \beta$ decay has also been studied for the other isotopes in the detector but only partial exposures have been published. The measurements of the $2 v \beta \beta$ half-life and the limits for $0 v \beta \beta$ decay in ${ }^{82} \mathrm{Se},{ }^{150} \mathrm{Nd},{ }^{96} \mathrm{Zr}$ and ${ }^{130} \mathrm{Te}$ are summarised in Tab. 3. The NEMO-3 collaboration is currently finalising the analysis of the full exposure for all the isotopes.

\begin{tabular}{|c|c|c|c|c|c|c|}
\hline Isotope & $\begin{array}{c}\text { Mass } \\
\mathrm{g}\end{array}$ & $\begin{array}{c}\text { Exposure } \\
\text { days }\end{array}$ & $\begin{array}{c}\mathrm{T}_{1 / 2}^{2 v} \times 10^{19} \mathrm{y} \\
\end{array}$ & $\begin{array}{c}\mathrm{T}_{1 / 2}^{0 v} \\
\text { y at } 90 \% \text { C.L. }\end{array}$ & $\begin{array}{c}\left\langle m_{v}\right\rangle \\
\text { eV at } 90 \% \text { C.L. }\end{array}$ & Reference \\
\hline${ }^{82} \mathrm{Se}$ & 932 & 389 & $9.6 \pm 1.0$ & $>1.0 \times 10^{23}$ & $<1.7-4.9$ & {$[8]$} \\
${ }^{150} \mathrm{Nd}$ & 37 & 924.7 & $0.90 \pm 0.07$ & $>1.8 \times 10^{22}$ & $<4.0-6.3$ & {$[12]$} \\
${ }^{96} \mathrm{Zr}$ & 9.4 & 1221 & $2.35 \pm 0.21$ & $>9.2 \times 10^{21}$ & $<7.2-19.5$ & {$[13]$} \\
${ }^{130} \mathrm{Te}$ & 454 & 1275 & $70 \pm 14$ & $>1.3 \times 10^{23}$ & $<1.4-3.5$ & {$[14]$} \\
\hline
\end{tabular}

Table 3: The $2 v \beta \beta$ half-life and the limits for $0 v \beta \beta$ decay in ${ }^{82} \mathrm{Se},{ }^{150} \mathrm{Nd},{ }^{96} \mathrm{Zr}$ and ${ }^{130} \mathrm{Te}$.

\section{The SuperNEMO experiment}

SuperNEMO is the next generation $0 v \beta \beta$ experiment based on the NEMO-3 technique combining tracking and calorimetry. The goal is to gain 2 orders of magnitude in the current half-life sensitivity $T_{1 / 2}^{0 v}>1 \times 10^{26}$ y corresponding to effective neutrino mass of $\left|m_{\beta \beta}\right|<0.04-0.10 \mathrm{eV}$. SuperNEMO will consist of 20 identical planar modules housing each $5 \mathrm{~kg}$ of double beta decay isotope (Fig. 7). The ${ }^{82} \mathrm{Se}$ is the baseline isotope given the ease to enrich and purify high masses by actual means in a reasonable time. Some developments are ongoing to obtain significant amounts of ${ }^{150} \mathrm{Nd}$ and ${ }^{48} \mathrm{Ca}$ which are of strong interest because of their high $\mathrm{Q}_{\beta \beta}$-value. The double beta decay sources will be shaped in thin foil made of selenium powder, polyvinyl-alcohol glue and thin mylar film.

A dedicated R\&D allowed to reduce the calorimeter energy resolution down to $4 \%$ FWHM at $3 \mathrm{MeV}$. A particular effort has been made to reduce the radon emanation of the material and to prevent external radon penetration in the detector volume. The most critical materials have been tested in radon emanation chambers coupled to very sensitive radon detectors. Radon adsorption materials are also being tested to improve the purification systems. The radio-purity of the source foils is being measured in the dedicated BiPo detector [20] able to reach a sensitivity of $2 \mu \mathrm{Bq} / \mathrm{kg}$ 


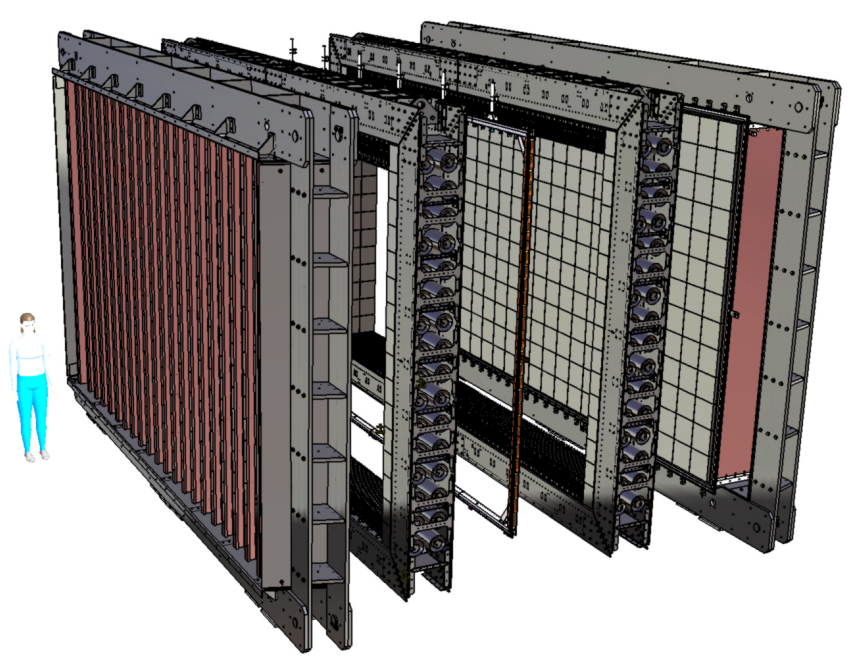

Figure 7: Exploded view of a SuperNEMO detection module. The source plane in the middle is surrounded by two tracker volumes and two calorimeters.

and $10 \mu \mathrm{Bq} / \mathrm{kg}$ in ${ }^{208} \mathrm{Tl}$ and ${ }^{214} \mathrm{Bi}$ respectively.

The collaboration is currently building the first module called demonstrator which will observe $7 \mathrm{~kg}$ of ${ }^{82} \mathrm{Se}$. The demonstrator aims to register no background events in the $0 v \beta \beta$ energy region after $2.5 \mathrm{y}$. This will validate the choice of the materials adopted to reduce the background contamination and will validate the physics potential of the full SuperNEMO detector. The half-life sensitivity of the demonstrator module is expected to be $T_{1 / 2}^{0 v}>6.5 \times 10^{24}$ y leading to a mass limit $\left|m_{\beta \beta}\right|<0.20-0.40 \mathrm{eV}$ (90\% C.L.) which improves by factor 6 the current limit obtained by NEMO-3 with ${ }^{100}$ Mo.

The demonstrator calorimeter is under construction with the assembly of the polystyrene scintillators with the 8" Hamamatsu PMTs. The pure iron mechanical structure and the PMT magnetic shields have been constructed and are also under assembly.

The first quarter of the tracker has been assembled and populated with the drift cells produced by the wiring robot and it is currently under commissioning at sea level. Preliminary radon emanation test results in an activity of $0.236 \pm 0.035 \mathrm{mBq} / \mathrm{m}^{3}$. The second quarter of the tracker is currently under assembly.

A total of $5.5 \mathrm{~kg}$ of ${ }^{82} \mathrm{Se}$ have been purchased and purified. The source fabrication materials have been selected and the two first strips of the source foils are under measurement in the BiPo detector. The calibrations systems (source deployment and light injection) have demonstrated the stability requirements. The calorimeter front-end digitising electronic boards have also been produced and are under test. The production of the tracker front-end electronic has started and the general control board prototype is in final tests. The integration of the demonstrator in the LSM is ongoing and the physics data taking is expected to start in 2016.

\section{Summary}

The NEMO-3 experiment was unique in the direct reconstruction of the full signature of 
$0 v \beta \beta$ decays with powerful background rejection capabilities. With an exposure of $34.3 \mathrm{~kg} \times \mathrm{y}$, no evidence for the $0 v \beta \beta$ decay of ${ }^{100}$ Mo has been found. Taking into account statistical and systematic uncertainties, the half-life limit for the light Majorana neutrino mass mechanism is $T_{1 / 2}^{0 v}>1.1 \times 10^{24}$ y (90 \% C.L.) which corresponds to a limit on the effective Majorana neutrino mass of $\left|m_{\beta \beta}\right|<0.33-0.62 \mathrm{eV}$. The absence of events in the high energy part of the spectrum is an encouraging result for future experiments based on the tracking+calorimetry technique planning to use high $\mathrm{Q}_{\beta \beta}$ isotopes such as ${ }^{48} \mathrm{Ca},{ }^{96} \mathrm{Zr}$ and ${ }^{150} \mathrm{Nd}$.

SuperNEMO is the next generation experiment and the first demonstrator module is currently under construction in the LSM. No background event is expected in the $0 v \beta \beta$ region in 2.5 years for $7 \mathrm{~kg}$ of ${ }^{82} \mathrm{Se}$. The half-life sensitivity is expected to be $T_{1 / 2}^{0 v}>6.5 \times 10^{24}$ y corresponding to an effective mass sensitivity $\left|m_{\beta \beta}\right|<0.2-0.4 \mathrm{eV}$ (90\% C.L.). Physics data taking of the demonstrator is expected to start in 2016 .

\section{References}

[1] J. Kotila and F. Iachello, Phys. Rev. C 85 (2012) 034316; S. Stoica and M. Mirea, Phys. Rev. C 88 (2013) 037303

[2] J. Hyvarinen and J. Suhonen, Phys. Rev. C 91 (2015) 024613; F. Šimkovic et al., Phys. Rev. C 87 (2013) 045501; J. Barea and F. Iachello, Phys. Rev. C 79 (2009) 044301; P. K. Rath et al., Phys. Rev. C 82 (2010) 064310; T.R. Rodriguez and G. Martinez-Pinedo, Phys. Rev. Lett. 105 (2010) 252503; J. Mendez et al., Nucl. Phys. A 818 (2009) 139.

[3] R.G.H. Robertson, Modern Phys. Lett. A 28, 1350021 (2013); S. Dell'Oro, S. Marcocci, and F. Vissani, Phys. Rev. D90, 033005 (2014).

[4] R. Arnold et al., Nucl. Inst. Meth. A 536 (2005) 79

[5] R. Arnold et al., Eur. Phys. J. C 70 (2010) 927

[6] J. Argyriades et al., Nucl. Inst. Meth. A 606 (2009) 449

[7] P. Domin et al. Nucl. Phys. A753 (2005) 337-363

[8] R. Arnold et al., Phys. Rev. Lett. 95 (2005) 182302

[9] R. Arnold et al., Phys. Rev. D 89 (2014) 111101(R)

[10] K.A. Olive et al., Particle Data Group, Chin. Phys. C 38, 090001 (2014).

[11] T. Junk, Nucl. Inst. Meth. A 434 (1999) 435

[12] R. Arnold et al., Phys. Rev. C 80 (2009) 032501

[13] R. Arnold et al., Nucl. Phys. A 847 (2010) 168

[14] R. Arnold et al., Phys. Rev. Lett. 107 (2011) 062504

[15] J. Suhonen, Nucl. Phys. A 700 (2002) 649; T. Tomoda, Rept. Prog. Phys. 54 (1991) 53; K. Muto, E. Bender and H.V. Klapdor, Z. Phys. A 334 (1989) 187

[16] A. Faessleret al., Phys. Rev. D 83 (2011) 113015

[17] R. Arnold et al., Nucl. Phys. A 765 (2006) 483

[18] M. Doi, T. Kotani, and E. Takasugi, Phys. Rev. C 37 (1988) 2104

[19] R. Arnold et al., Nucl. Phys. A 781 (2007) 209

[20] H. Gomez, Nucl. Instrum. Meth. A 718 (2013) 52 